\title{
NUMERICAL AND EXPERIMENTAL ANALYSIS OF TURBULENT FLUID FLOW AROUND LATEST GENERATION CYCLING FRAME
}

\author{
M. CASTELLINI ${ }^{1}$, M. BARBANERA ${ }^{1}$, M. SCUNGIO ${ }^{1} \&$ F. ARPINO ${ }^{2}$ \\ ${ }^{1}$ Department of Economics, Engineering, Society and Business, University of Tuscia, Italy. \\ ${ }^{2}$ Department of Civil and Mechanical Engineering, University of Cassino and Southern Lazio, Italy.
}

\begin{abstract}
Computational fluid dynamics (CFD) is a branch of fluid mechanics that uses numerical analysis and data structures to analyse and solve problems that involve fluid flows. Today, CFD plays a decisive role in the cycling industry, which affects not only bicycle manufacturers, but also, above all, bicycle component suppliers. In fact, aerodynamic research takes place not only in the cyclist's best riding position, but also in the design of the components and frames that make up a racing bike. The frame design is essential both for its ability to oppose the aerodynamic resistance and to adapt the cyclist to the best geometry. Among the multiple outlets of the method, the simulation of external aerodynamic flows shows a fundamental importance for the understanding of the role played by the design of the bicycle. Once a numerical analysis was set correctly, it was then possible to predict with good reliability the fluid dynamic behaviour of an entire structure without the need to use experimental approaches every time. The main aim of this study consists of the validation of a numerical model through experiments conducted on a scale model of a latest generation cycling frame in an open chamber wind tunnel by means of the Particle Image Velocimetry (PIV) technique. In particular, the scale model used was investigated in two specific regions. The experimental data were compared to numerical results obtained employing turbulence model, and the validated numerical tool was subsequently applied to estimate the drag coefficient of two different types of handlebars (aerodynamic and standard versions). The standard cylindrical handlebar folds were replaced by products made of composite and with the most innovative and modern shapes, able to significantly reduce the aerodynamic resistance values. Indeed, in the design phase, the measurement of the drag coefficient is a fundamental procedure. As expected, the presence of aerodynamic profiles generated a low drag coefficient, one of the most important aerodynamic conditions.

Keywords: computational fluid dynamics, cycling frame, drag coefficient, turbulence model, Particle Image Velocimetry.
\end{abstract}

\section{INTRODUCTION}

One of the most important results of aerodynamic is the determination of the aerodynamic forces acting on a body, which therefore alter its range of motion. The fluid dynamic effects can be simplified by the decomposition in two directions (parallel and orthogonal to the motion) of a resulting force. The component parallel to the motion, called drag, plays a dominant role in cycling applications because it is the main opposing force that a cyclist has to overcome when cycling on level ground at moderate-to-high speeds. Therefore, the aerodynamic study of the bike-cyclist set is identified as a key factor for the analysis and improvement of performance.

The resistance of a body immersed in the fluid originates from the wake flow generated by the body itself. A blunt and irregular body disturbs the air flowing around it, forcing the air to separate from the body's surface. This particular condition generates a difference in pressure between the front and the back regions of the body [14]. The location at which the flow separates determines the size of the separation zone, and consequently the drag force [6]. Streamlined designs help the air close more smoothly around these bodies and reduce pressure drag. 
The formation of the wake stream is an extremely complex condition to be quantified from a mathematical perspective. The rigorous analytical characterization of the fluid dynamic problem must, therefore, be effectively introduced within appropriate software for achieving the desired solution. Computational fluid dynamic (CFD) analysis represents a powerful tool for the evaluation of cycling aerodynamics because it can provide a large amount of information, difficult to obtain with experimental measurements [2]. This study shows that the CFD technique, with its capability for testing a wide range of geometries at a relatively low cost, may be used to complement experimental tests. From a practical point of view, the reproduction, in CFD simulations, of the turbulent behaviour of the flow constitutes the most critical step of the entire modelling process.

Nowadays, the importance of aerodynamics in cycling is increasingly recognized by both engineers and cyclists. There is an abundance of published literature focusing on the development of more aerodynamically efficient rider positions, racing frames and components. Although the physical presence of the athlete symbolizes the prevalent contribution to the aerodynamic resistance [8], the suggested experimental tests refer exclusively to the material components (frame and handlebar). Most recent designs are concentrated on shifting from round tubes to oval or tear-shaped tubes [15]. There is a delicate balancing act between maintaining a good strength-to-weight ratio while improving aerodynamic efficiency. It was also found that the handlebars contributed to $10 \%$ of the drag on the frame [15]. By measuring the traction resistance and also the energy cost of cycling per unit distance [5], the aerodynamic benefit of riding a bicycle with an aerodynamically optimized frame was studied. Measurements were recorded by towing two riders around a velodrome. It was found that a bike with an aerodynamic frame would travel approximately $3 \%$ faster than a bike with a traditional frame. CFD analyses were recently used to study the flow around a mountain bike and rider [10]. The investigation showed that as far as aerodynamic drag is concerned, the rider contributes $64 \%$, the forks $7 \%$ and the handlebar, front wheel and frame all individually contribute approximately $6.5 \%$.

The aim of the present work consists of the numerical and experimental investigation of the aerodynamic behaviour of a last generation cycling frame. To this aim, averaged velocity field measurements were conducted on a 1:27 scaled model, employing an open chamber wind tunnel and the Particle Image Velocimetry (PIV) technique, already applied in other previous studies $[12,3]$. The experimental tests, conducted on two specific regions, were then used to validate $3 \mathrm{D}$ numerical simulations. In this way, it is then possible to predict with good reliability the fluid dynamic behaviour of an entire structure without the need to use experimental approaches every time. In particular, the aerodynamic performance of two different types of handlebars was studied in detail.

\section{METHODS}

\subsection{The cycling frame}

Figure 1 shows the 3D model of the cycling frame considered for experimental investigations. The small size of the wind tunnel measurement chamber required the use of a scale model; specifically, a 1:27 scale moulding was planned, which has $273 \mathrm{~mm}$ of length, 114 $\mathrm{mm}$ of width and $188 \mathrm{~mm}$ of height.

The realization of an excellent surface finish, one of the most important requirements for an aerodynamic characterization, presupposes different moulding method compared to conventional plastic techniques of fused deposition modelling $3 \mathrm{D}$ printer. 

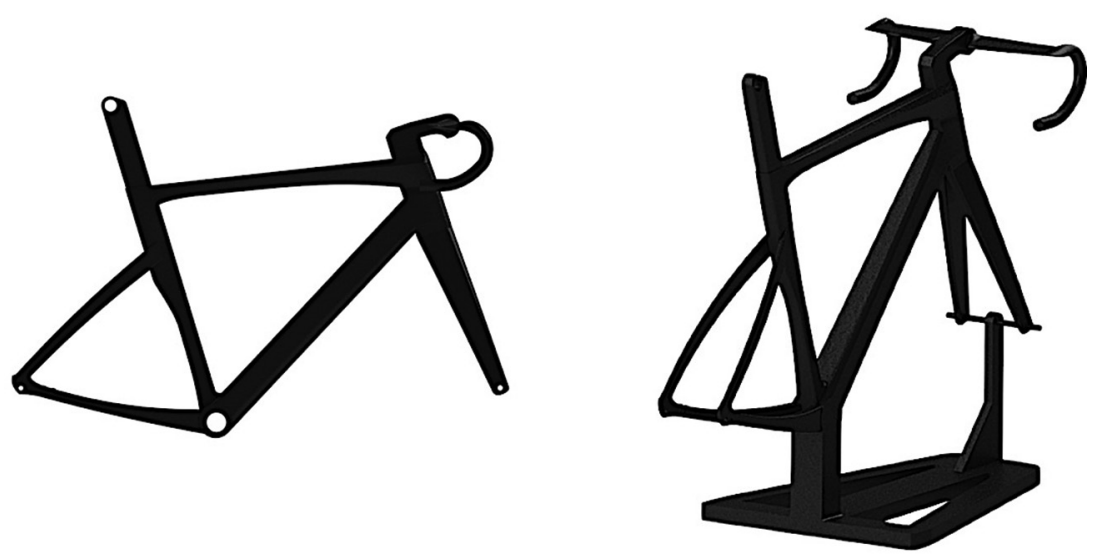

Figure 1: 3D model of the cycling frame.

The model was made with Sharebot Big format 3D resin printer; its big print volume allows to create aesthetic and functional prototypes of large dimensions in a single piece. For moulding, the Sharebot Big uses photosensitive resins (Lumi React Hard and Detailed) with Led Screen Light (LSL) technology, able to create resistant objects and prototypes. The scale model was used for experimental investigations in the wind tunnel, employing the PIV technique, and the experiments were numerically reproduced by means of 3D CFD simulations.

\subsection{Wind tunnel and PIV system setup}

The experimental investigations were performed in the wind tunnel of the Laboratory of Industrial Measurements (LaMI) of the University of Cassino and Southern Lazio (Italy). In particular, the wind tunnel used belongs to the closed circuit type, in which the air circulation is generated by a fan fitted on the shaft of a synchronous motor powered by a static converter (inverter). The synchronous motor-inverter system determines a precise adjustment of the fan rotation velocity and, therefore, of the air velocity in the tunnel. The acceleration of the flow is caused by the presence of a convergent duct located in the inlet section.

The PIV technique is based on a simple principle: the flow is seeded by micron-sized particles and is illuminated twice by a laser light sheet in a very short time interval. Two images are obtained, from a special camera, when the particles are illuminated, and the local velocity vectors reconstruction is obtained from the two images over different interrogation areas via cross-correlation method [7]. In order to compensate the blockage effect of the wind tunnel [12], it was necessary to adjust the air velocity used as input in the later CFD analyses. The air velocity imposed at the beginning of each test, being in fact influenced by the physical presence of the scale model, could not be considered as the reference value for numerical analyses.

In Fig. 2, it is possible to observe the positioning of the laser beam with the corresponding regions of investigation where details of the micron-sized particles are visible at the back of the model.

In Table 1, the main experimental test parameters are resumed for the reference wind velocity investigated. Detailed discussion about the significance of the parameters reported is available in literature [4]. 

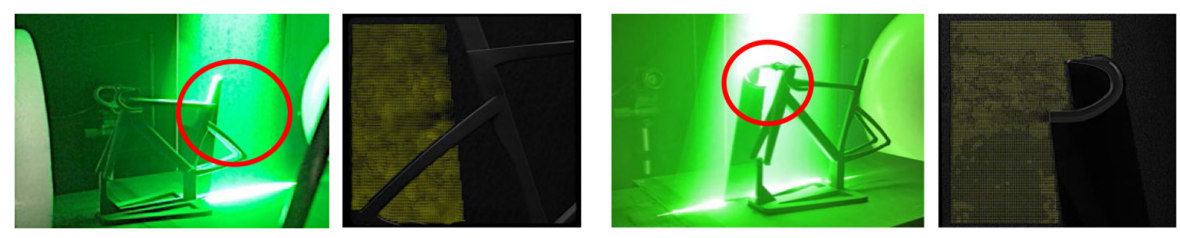

Figure 2: Measurement domains adopted for the PIV analysis of the wake flow in the wind tunnel.

Table 1: Main test parameters of PIV measurements.

\begin{tabular}{|c|c|}
\hline Uniform flow velocity & $19.90 \mathrm{~m} / \mathrm{s}$ \\
\hline Target flow & 2D air flow \\
\hline Measurement facility & Open chamber wind tunnel \\
\hline Measurement area & $200 \mathrm{~mm} \cdot 200 \mathrm{~mm}$ \\
\hline $\mathrm{T}_{\mathrm{REF}}$ & $28^{\circ} \mathrm{C}$ \\
\hline Bicycle width & $188 \mathrm{~mm}$ \\
\hline Bicycle length & $273 \mathrm{~mm}$ \\
\hline \multicolumn{2}{|c|}{ Calibration } \\
\hline Distance of reference points & $120 \mathrm{~mm}$ \\
\hline Distance of reference image & 1700 pixels \\
\hline Magnification factor & 14.17 pixels $/ \mathrm{mm}$ \\
\hline \multicolumn{2}{|c|}{ Flow visualization } \\
\hline Tracer generator & $\begin{array}{c}\text { Stage-smoke generator (water-glycol } \\
\text { droplets) }\end{array}$ \\
\hline 1-min average particle diameter (mode) & $1.2 \mu \mathrm{m}$ \\
\hline Light source & Double pulse laser \\
\hline Max. laser power & $135 \mathrm{~mJ}$ at $535 \mathrm{~nm}$ \\
\hline Thickness of the laser light sheet & $\cong 1 \mathrm{~mm}$ \\
\hline Time interval & $20.5 \mu \mathrm{s}$ \\
\hline \multicolumn{2}{|c|}{ Image detection } \\
\hline Camera resolution & $2048 \cdot 2048$ pixels \\
\hline Sampling frequency & $7.25 \mathrm{~Hz}$ \\
\hline Distance from target & $0.58 \mathrm{~m}$ \\
\hline \multicolumn{2}{|c|}{ Data processing } \\
\hline Pixel unit analysis & Cross-correlation method \\
\hline Correlation area size & 3030 pixels \\
\hline
\end{tabular}




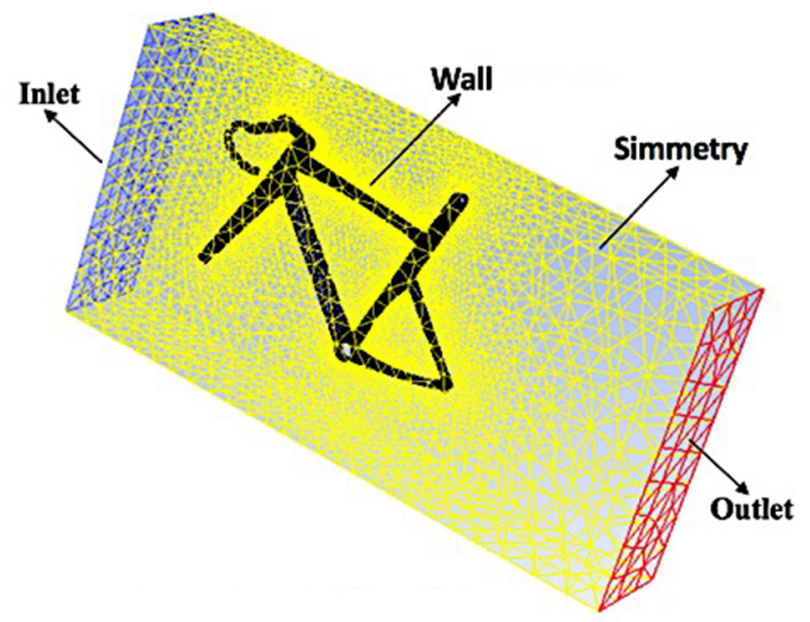

Figure 3: Computational domain for the numerical simulations of the 1:27 scale model of the cycling frame and the boundary conditions used.

\subsection{Mathematical and numerical models}

The numerical approach of the fluid dynamic distribution of the flow around the cycling frame involved the use of the standard turbulence model solved with the commercial software Ansys Fluent [9, 11].

In Fig. 3, the computational domain and the boundary conditions employed are presented. A perfect geometric symmetry is an essential condition for the subsequent reduction of computational nodes; in this case, only one-half of the domain from the symmetry plane was considered. The extension of the external domain is an extremely important parameter because it heavily conditions the final results. It is usually a good rule that the dimensions contain most of the natural development of the fluid dynamic effects. At the inlet, the x-component of the velocity was imposed, reproducing the same velocity profile as obtained from the PIV measurement, considering the blockage effect of the wind tunnel. The outlet was placed at a distance of $350 \mathrm{~mm}$ from the back of the model, while on the model surface, a wall boundary condition was imposed (zero velocity condition). A symmetry condition was imposed on all the other sides. A last consideration concerns the roughness of the material which can influence the flow field of the fluid particles. In these simulations, the problem was simplified with a zero 'wall roughness'.

To accurately describe the flow at an obstacle, specific dimensionless reference parameters are used, such as the distance from the wall [1]. The value of the dimensionless distance is indispensable for understanding the different regions of the turbulent boundary layer. Indicators such as the drag coefficient require a detailed study of the phenomenology established near the surface (viscous sub-layer) of the body under examination. In other words, it was necessary to provide a correct positioning of the first computational node of the mesh (, first layer thickness) through a particular localized thickening of the mesh (inflation). This function reproduced, on the basis of the value of the first thickness layer, a predetermined and structured series of layers immediately after the body (Fig. 4). 


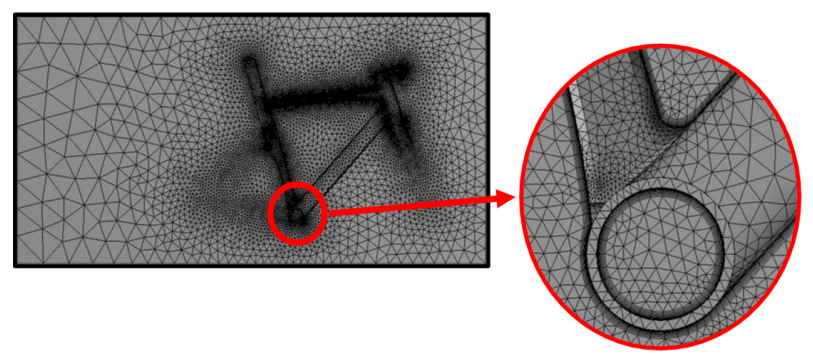

Figure 4: Mesh detail (inflation).

Starting from inflation, a grid refinement was made on the external domain using 10 layers and a minimum grid size of $0.2 \mathrm{~mm}$ with a growth rate of 1.10. A slow transition allowed the creation of a very detailed grid near the body. A fine span angle centre was used to generate condensations that perfectly adapt to the structural contours and consequently do not cause unpleasant geometric distortions in reproduction. The resulting unstructured computational grid was composed of about 1 million of tetrahedral elements.

The reliability of the final results is heavily influenced by a variety of factors. To quantify the robustness of the numerical problem, a sensitivity analysis of the grid was done by varying the first layer thickness . For practical matters (limited computational resources), the sensitivity analysis of the grid was conducted exclusively on the handlebar by monitoring the drag coefficient and evaluating the velocity profiles in specific regions. The optimal configuration found $\left(y_{1}=7.5 \cdot 10^{-5} \mathrm{~m}\right)$ was then used for the analysis of the entire structure.

A simple scheme was used for the pressure-velocity. The spatial discretization was instead performed with first-order algorithms.

\subsection{Drag coefficient}

The most important parameter in aerodynamic cycling studies is the drag force, , which is the force in the longitudinal flow direction, opposite to the bicycle movement.

Dimensionless drag coefficient could be defined as follows:

$$
y_{1}=7.5 \cdot 10^{-5} \mathrm{~m}
$$

where $\rho(\mathrm{kg} /)$ is the air density, $v(\mathrm{~m} / \mathrm{s})$ is the air velocity and $\mathrm{A}\left(\mathrm{m}^{2}\right)$ is the reference front model surface. The value of the drag force derives from the linear combination of two different contributions: pressure force, which is due to a pressure difference across the surface, and viscous force, which is due to the friction acting in the opposite direction of the flow.

\section{RESULTS AND DISCUSSION}

\subsection{PIV-CFD comparison}

The experimental data obtained with PIV was compared to the numerical results; two specific regions were subjected to the comparison procedure. The PIV results, derived from the acquisition of 750 images appropriately combined in the post-processing phase, represent a $2 \mathrm{D}$ distribution of average quantities. For this reason, CFD analyses also reproduce a steady state. The velocity of $19.90 \mathrm{~m} / \mathrm{s}$ detected at the entrance of the wind tunnel was affected by 


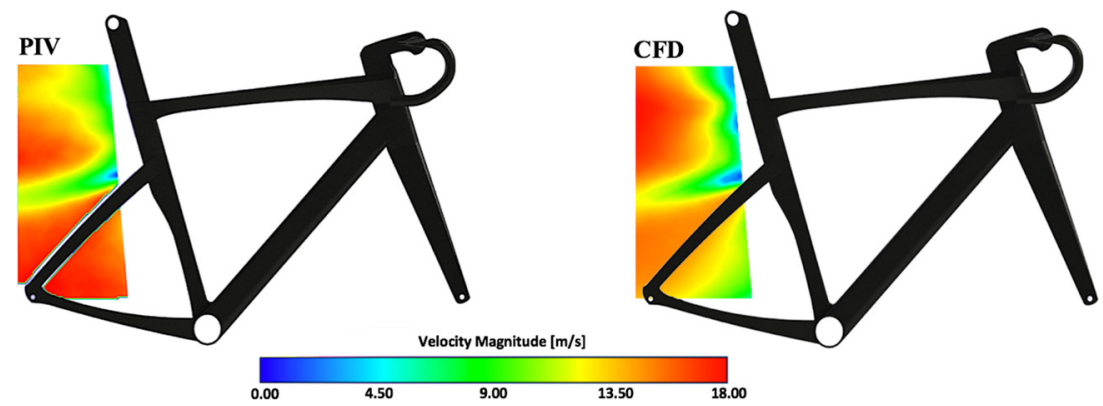

Figure 5: PIV and CFD (first region investigated): velocity distribution with reference flow velocity of $21 \mathrm{~m} / \mathrm{s}$.

the presence of the model and, therefore, could not be considered as the input reference value for CFD simulation. From the experimental results, a velocity of $21.00 \mathrm{~m} / \mathrm{s}$ was measured in undisturbed conditions; this value was assigned as input in Ansys Fluent.

The first region considered includes the wake flow present on the plane of symmetry and evaluated behind the seat post. Figure 5 shows a purely qualitative distribution of velocity evaluated through the PIV and CFD analyses on the investigation plan chosen. Both techniques show similar kinematic behaviours in the upper region of the domain. In the lower region of the investigation plan, CFD analyses identify a less-intense phenomenology compared to PIV technique. Therefore, in numerical results, there is an attenuation of the flow in the areas adjacent to the frame, which increases progressively on going down.

In broad terms, a similar distribution of turbulent kinetic energy is also evident (Fig. 6). The PIV is able to highlight a large extension of the turbulent phenomenon in most of the study domain. CFD analyses show the highest value of turbulent kinetic energy, but, at the same time, moderate phenomena of recirculation in the portion included between the rear frames compared to PIV technique.

The considerations previously reported regarding the qualitative distribution of turbulent kinetic energy are accompanied by analytical trends evaluated at two specific locations (Fig. 7).

The second investigation region is represented by a plane passing through the symmetry line of the handlebar bend. The acquisition methods make the PIV technique limited to displaying the wake effects only, in effect generating no information about the region in direct contact with the handlebar bend (shadow zone).

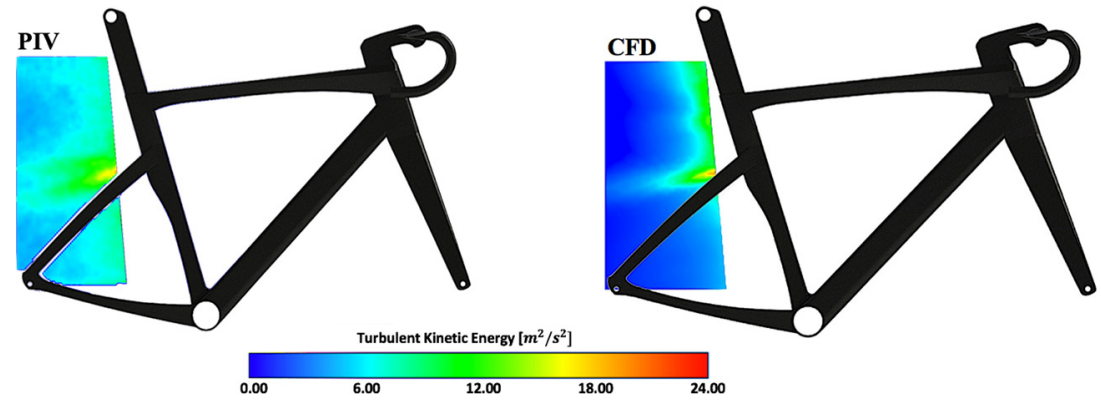

Figure 6: PIV and CFD (first region investigated): turbulent kinetic energy distribution with reference flow velocity of $21 \mathrm{~m} / \mathrm{s}$. 

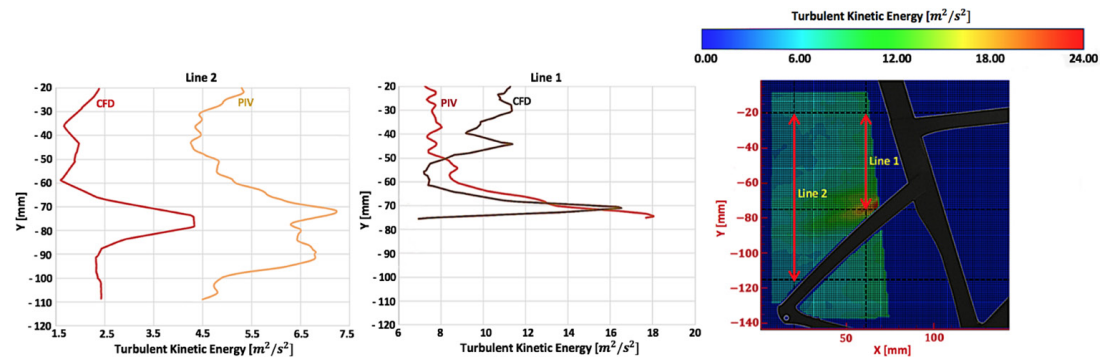

Figure 7: Turbulent kinetic energy profiles evaluated at the lines 1 and 2, with reference flow velocity of $21 \mathrm{~m} / \mathrm{s}$ : PIV and CFD in comparison.

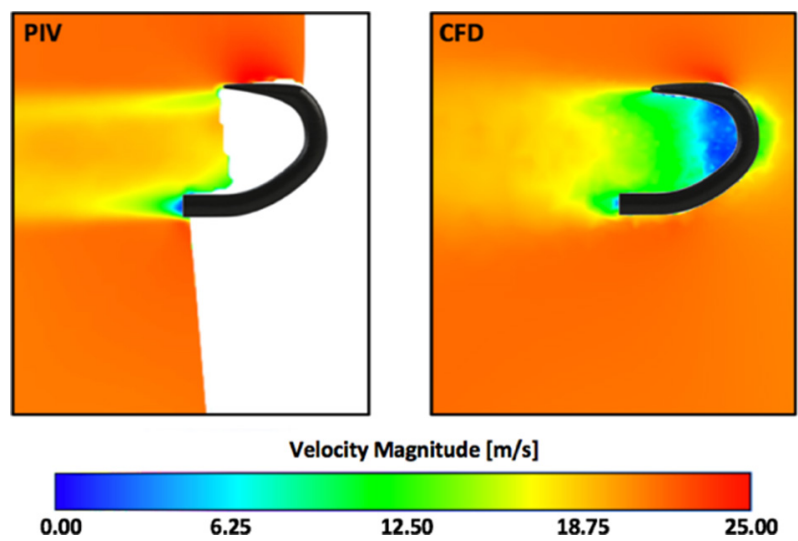

Figure 8: Velocity magnitude profiles evaluated at the line, with reference flow velocity of 21 $\mathrm{m} / \mathrm{s}$ : PIV and CFD in comparison.
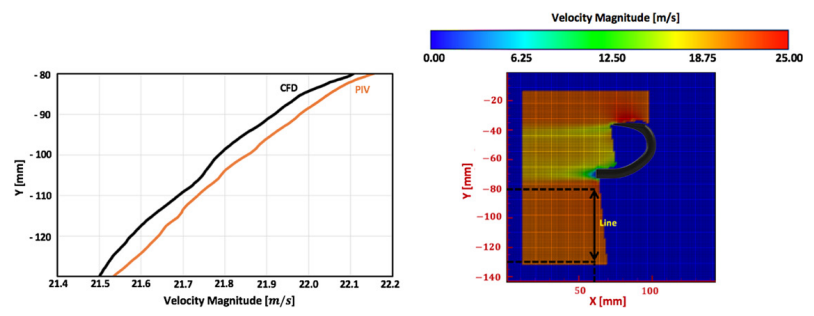

Figure 9: PIV and CFD (second region investigated): velocity distribution with reference flow speed of $21 \mathrm{~m} / \mathrm{s}$.

CFD analyses reproduce, similar to PIV, the undisturbed flow conditions in the lower part of the measurement domain (Figs. 5 and 8) and similar kinematic behaviours after the handlebar bend (Fig. 9). However, like in the first test (Fig. 5), it is possible to observe that CFD is more influenced by the physical presence of an obstacle.

The regions most affected by the effects of turbulence are the same in both techniques adopted, even if the CFD denotes a lesser extension of the phenomenology (Fig. 10).

In conclusion, on the basis of the qualitative and quantitative comparisons proposed above, the CFD analyses reproduce with a good approximation the experimental results obtained 

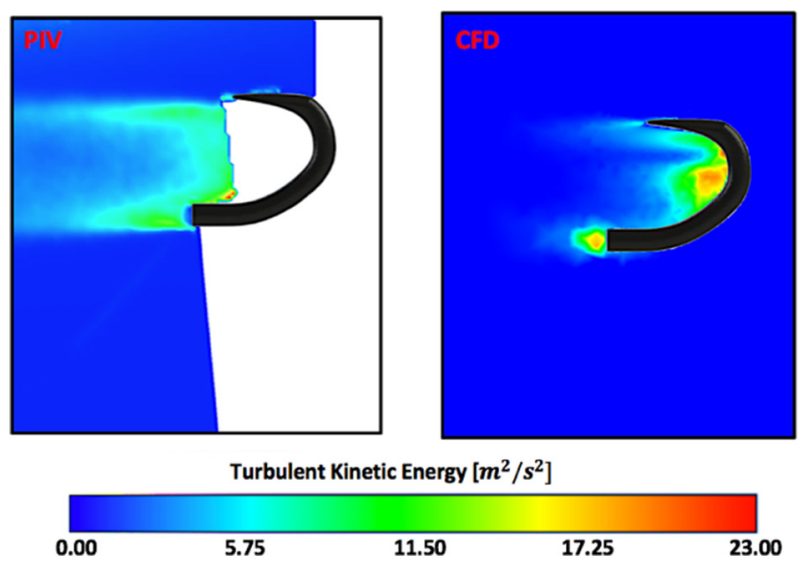

Figure 10: PIV and CFD (second region investigated): turbulent kinetic energy distribution with reference flow velocity of $21 \mathrm{~m} / \mathrm{s}$.

with the PIV technique. The difference found can be led to use of first-order algorithms for spatial discretization, available in the pressure-based and density-based solvers.

\subsection{Drag coefficient evaluation}

Once the numerical analyses were validated by the PIV measurements, it was possible to carefully examine the aerodynamic behaviour, in terms of drag coefficient, of two different types of handlebars. In particular, the aerodynamic configuration present in the model used for validation (Fig. 11b) was compared with a standard type composed of cylindrical tubulars (Fig. 11a). The lack of reference data was imposed the comparison with a now obsolete type of handlebar. In this way, it was possible to establish the aerodynamic potential of the considered products and then to attribute an explanation to their values.

As expected, numerical analyses show that an aerodynamic handlebar generates a lower drag coefficient than a standard configuration (Table 2). This substantial difference derives from the intensity of the pressure forces, which are due to a pressure difference across the structure. The aerodynamic profiles are designed to significantly reduce the turbulent effects of the flow behind the body and thus minimize its resistance when immersed in a viscous fluid. The viscous forces evaluated along the flow direction, due to the friction generated during the relative motion between the body and the external fluid, are perfectly comparable in both handlebars.

The different aerodynamic behaviours are conveniently visible in the post-processing reconstructions shown in Fig. 12 where the turbulent kinetic energy and the velocity distribution are evaluated on a generic middle plan (Fig. 13). A more complex geometry is able to
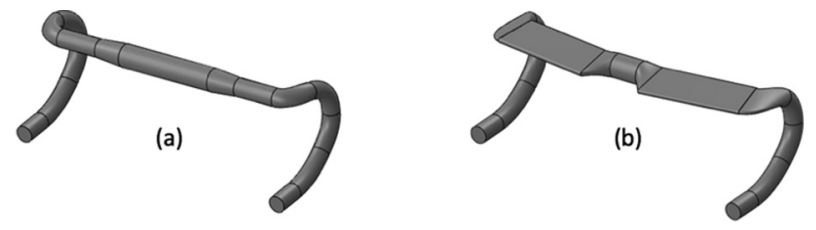

Figure 11: Standard (a) and aerodynamic (b) handlebars. 
Table 2: Aerodynamic parameters evaluated with a reference flow velocity of $15 \mathrm{~m} / \mathrm{s}$.

\begin{tabular}{llll}
\hline Handlebar type & Pressure force $(\mathbf{N})$ & Viscous force $(\mathbf{N})$ & Drag coefficient $\mathbf{C}_{\mathbf{D}}$ \\
\hline Standard & 0.348 & 0.040 & 0.280 \\
Aerodynamic & 0.206 & 0.045 & 0.161 \\
\hline
\end{tabular}

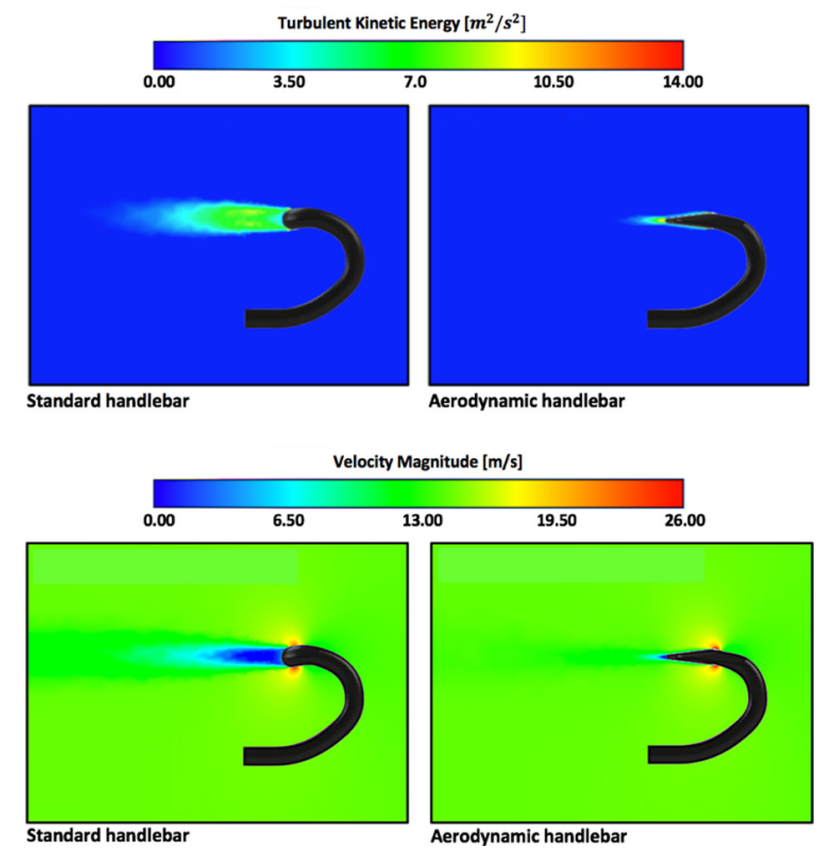

Figure 12: CFD analysis on the two different types of handlebars. Turbulent kinetic energy and velocity distribution evaluated on a middle plan with a reference flow speed of $15 \mathrm{~m} / \mathrm{s}$.
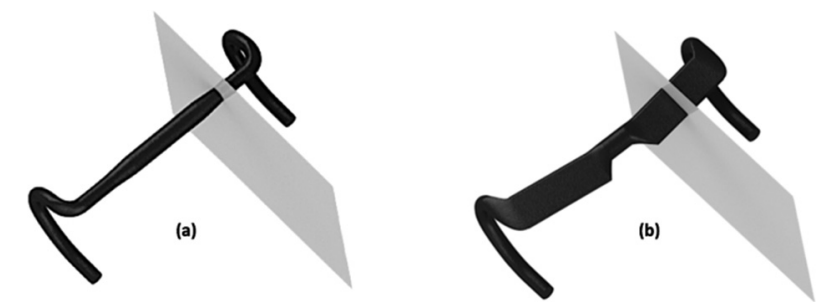

Figure 13: Reference planes on the standard (a) and the aerodynamic (b) handlebar.

perfectly convey the flow lines immediately after passing the body. This scenario minimizes the undesirable formation of recirculation phenomena.

Table 3 shows the influence of velocity on the drag force and, therefore, on the drag coefficient of the aerodynamic handlebar. Although a speed increase determines higher pressure and viscous forces, there is a reduction in the drag coefficient. This trend is analytically justified by the structure of the eqn (1), where there is a quadratic dependence with speed. In other words, the influence of speed is much higher than the progressive increase of the drag force. 
Table 3: Velocity influence on aerodynamic parameters.

\begin{tabular}{llll}
\hline Speed $(\mathbf{m} / \mathbf{s})$ & Total pressure force $(\mathbf{N})$ & Total viscous force $(\mathbf{N})$ & ${\text { Drag coefficient } \mathbf{C}_{\mathbf{D}}}$ \\
\hline 10.0 & 0.103 & 0.020 & 0.178 \\
12.5 & 0.150 & 0.032 & 0.168 \\
15.0 & 0.206 & 0.045 & 0.161 \\
17.5 & 0.271 & 0.061 & 0.157 \\
\hline
\end{tabular}

\section{CONCLUSIONS}

In order to verify the suitability of the numerical model, results from turbulence model, in terms of speed and turbulent kinetic energy, were compared with experimental data from a wind tunnel investigation made out with PIV technique. To obtain the experimental results on the cycling frame, it was necessary to use a 3D scale model printed in resin and therefore characterized by a high surface finish. In detail, two regions of particular interest were studied in order to understand the flow wake effects and the behaviour of some fluid dynamic properties. Referring to the results discussed, it is evident that numerical simulations reproduce similar fluid dynamic phenomena compared to experimental tests.

The most significant differences observed can be attributed to the use of first-order algorithms for spatial discretization, available in the pressure-based and density-based solvers.

The validated numerical tool was applied to evaluate the drag coefficient of two different types of handlebars and to estimate their aerodynamic behaviour. Numerical analyses show how a racing handlebar has a lower drag coefficient than a standard configuration; considering a reference flow velocity of $15.0 \mathrm{~m} / \mathrm{s}$, the aerodynamic handlebar has a drag coefficient equal to 0.161 against 0.280 of the standard configuration. This result is closely related to its particular aerodynamic geometry designed to minimize aerodynamic drag during a competitive performance.

\section{REFERENCES}

[1] Abdellah, E. \& Wang, B., IOP Conference Series: Materials Science and Engineering, Volume 231, Issue 1, pp. 012173, 2017.

[2] Arpino, F., G. Cortellessa, Dell'Isola, M., Massarotti, N. \& Mauro, A. High order explicit solutions for the transient natural convection of incompressible fluids in tall cavities. Numerical Heat Transfer; Part A: Applications, 66(8), pp. 839-862, 2014. https://doi.org/10.1080/10407782.2014.892389

[3] Arpino, F., Cortellessa, G., Frattolillo, A., Caschera, M. \& Pelliccio, A., Experimental and numerical investigation of the effects of wind exposure on historical towns. Energy Procedia, 133, pp. 312-326, 2017. https://doi.org/10.1016/j.egypro.2017.09.394

[4] Bhattacharya, S., Charonko, J.J. \& Vlachos, P.P., Particle image velocimetry (PIV) uncertainty quantification using moment of correlation (MC) plane. Measurement Science and Technology, 29(11), article number 115301, 2018. https://doi.org/10.1088/13616501/aadfb4

[5] Capelli, C., Rosa, G., Butti, F., Ferretti, G., Veicsteinas, A. \& di Prampero, P., Energy cost and efficiency of riding aerodynamic bicycles. European Journal of Applied Physiology, 67(1), pp. 144-149, 1993. https://doi.org/10.1007/bf00376658

[6] Guilmineau, E., Computational study of flow around a simplified car body. Journal of Wind Engineering and Industrial Aerodynamics, 96(6-7), pp. 1207-1217, 2008. https:// doi.org/10.1016/j.jweia.2007.06.041 
[7] Keane, R.D. \& Adrian, R.J., Theory of cross-correlation analysis of PIV images. Applied Scientific Research, 49(1992), pp. 191-215, 2006. https://doi.org/10.1007/bf00384623

[8] Kyle, C.R. \& Burke, E.R., Improving the racing bicycle. Mechanical Engineering, 106(9), pp. 34-35, 1984.

[9] Launder, B.E. \& Spalding, D.B., The numerical computation of turbulent flows. Computer Methods in Applied Mechanics and Engineering, 3(2), pp. 269-289, 1974. https:// doi.org/10.1016/0045-7825(74)90029-2

[10] Lukes, R.A., Hart, J.H., Chin, S.B. \& Haake, S.J., The aerodynamics of mountain bicycles: The role of computational fluid dynamics. In 5th International Conference on the Engineering of Sport, Vol. 1 (Eds., Hubbard, M., Mehta, R. D. \& Pallis, J. M.) U.C. Davis, U.S.A., pp. 104-110, pp. 2004.

[11] Neft, I., Scungio, M., Culver, N. \& Singh, S., Simulations of aerosol filtration by vegetation: Validation of existing models with available lab data and application to nearroadway scenario. Aerosol Science and Technology, 50(9), pp. 937-946, 2016. https:// doi.org/10.1080/02786826.2016.1206653

[12] Scungio, M., Arpino, F., Profili, M., Rotondi, M., Focanti, V. \& Bedon, G., Wind tunnel testing of scaled models of a newly developed Darrieus-style vertical axis wind turbine. European Wind Energy Association Annual Conference and Exhibition 2015, EWEA 2015 - Scientific Proceedings.

[13] Tsubokura, M., Kobayashi, T., Nakashima, T., Nouzawa, T., Nakamura, T., Zhang, H., Onishi, K. \& Oshima, N., Computational visualization of unsteady flow around vehicles using high performance computing. Computers and Fluids, 38(5), pp. 981-990, 2009. https://doi.org/10.1016/j.compfluid.2008.01.020

[14] Ucho, W.H. \& Sovran, G., Aerodynamics of road vehicles. Annual Review of Fluid Mechanics, 25(1), pp. 485-537, 1993. https://doi.org/10.1146/annurev.fl.25.010193. 002413

[15] Zdravkovich, M.M., Aerodynamics of bicycle wheel and frame. Journal of Wind Engineering \& Industrial Aerodynamics, 40(1), pp. 55-70, 1992. https://doi.org/10. 1016/0167-6105(92)90520-k 